\title{
Was sich zeigt, wenn wir nicht mehr $d a$ sind: Über die Frage von Präsenz ${ }^{1}$
}

\section{Clara Arnold}

\section{Einleitung}

$\mathrm{Ob}$ es um systemrelevante Berufsgruppen und ihre oft prekär Beschäftigten geht, die in ihrer Arbeit nicht auf körperliche Anwesenheit verzichten können, um die Entscheidung darüber, wer zum Nahkreis der Personen gehört, zu denen noch weiterhin körperlicher Kontakt besteht, oder aber darum, dass physical distancing zu einer der wichtigsten Strategien zur Eindämmung des Virus wurde: Mit Blick auf die Ereignisse und Veränderungen rund um die globale Corona-Pandemie scheint sich die Frage nach Präsenz neu zu stellen.

Die Frage, wann sich Menschen wie anstecken können, ist sowohl eine epidemiologische und politische als auch eine räumliche. ${ }^{2}$ Auch die Hochschulen mussten darauf ihre eigenen Antworten finden: So wird bereits seit Mitte März 2020 an einem Status quo im Digitalen gearbeitet, der auf absehbare Zeit einen reibungslosen Semesterablauf und die Erbringung von Leistungen ermöglichen soll. Auf den ersten Blick scheint sich die Bedeutung der körperlichen Anwesenheit vor allem für diejenigen Studienfächer zu zeigen, die unweigerlich auf den Ort der Hochschule angewiesen sind. Doch die Relevanz von Anwesenheit zeigt sich auch für jene Fächer oder Disziplinen, die vergleichsweise leichter ins Digitale übertragen werden können - wenn auch vor einem anderen Hintergrund. Durch die vermeintlich problemlose Verlagerung zeigt sich die Bedeutsamkeit von Anwesenheit weniger und gerade deshalb umso existenzieller.

Obgleich diese Differenzierung für die politische Auseinandersetzung darüber, wer wann und wie an die Hochschulen zurückkehren darf, höchstwahrscheinlich relevant sein wird, rückt der vorliegende Beitrag die

1 Für wertvolle Kommentare danke ich Sofia Leikam und Peter Schuller.

2 Darauf wurde bereits vielfach hingewiesen: Hier möchte ich im Besonderen das Glossar zur Corona-Krise Kritische Theorien in der Pandemie (2020) des Frankfurter Arbeitskreises nennen. 
viel grundlegendere Frage nach Präsenz in den Mittelpunkt. ${ }^{3}$ Ausgehend von der Beobachtung, was sich zeigt, wenn wir nicht mehr $d a$ sind, möchte ich sowohl das Phänomen der Präsenz als auch die in ihm liegenden politischen Dimensionen betrachten. Dafür halte ich es für gewinnbringend, drei unterschiedliche theoretische Perspektiven heranzuziehen: erstens die Leibphänomenologie, die den Weltzugang als leiblichen Zugang beschreibt; zweitens die politische Theorie Judith Butlers, die zu einem großen Teil dazu beigetragen hat, die Strukturen offenzulegen und zu problematisieren, die das „Recht auf Erscheinen“ (Butler 2016) konstituieren; und drittens die Kritik des Theoretikers Alex Demirović, mit der sich die technokratische Transformation an Hochschulen als Steuerung von Präsenz begreifen lässt.

\section{Präsenz als Weltverhältnis}

Die fehlende Anwesenheit Anderer scheint schon fast eine banale Beobachtung in der aktuellen Corona-Pandemie zu sein. Wir sehen uns damit konfrontiert, die Welt durch Sehen oder Hören, ohne die körperliche Präsenz der Anderen, wahrzunehmen. Dabei konstatieren bereits erste Stimmen, dass "Gesellschaft auch ohne anwesende Körper funktioniert“ (Dickel 2020) - dass wir längst die mediale Sozialität leben. Die darin zutage tretende Möglichkeit der Flexibilität, nicht zwangsweise an einem Ort anwesend sein zu müssen, ermöglichte überhaupt erst die Durchführung des Sommersemesters 2020.

Im Gegensatz zu dieser funktionalistischen Betrachtung des Digitalen entwickeln der französische Philosoph Maurice Merleau-Ponty in seinem Hauptwerk Phänomenologie der Wabrnehmung (1966) und dessen Schüler, der Phänomenologe Bernhard Waldenfels in Das leibliche Selbst (2000), einen umfassenden Blick auf Wahrnehmung und die in ihr liegende Bedeutung von leiblicher Präsenz. Ihre theoretischen Perspektiven gründen sich auf mehrere Einwände, die an dieser Stelle dafür sensibilisieren sollen, warum die digitale Aufmerksamkeit die leibliche Anwesenheit nicht ersetzen kann.

3 Aufgrund eines offenen Briefes (2020), der die Präsenzlehre verteidigt und den zahlreiche Wissenschaftler"innen unterzeichnet haben, entfaltet sich seit Juni 2020 auch eine öffentliche Debatte über die Rolle von Präsenzlehre. Dazu nehmen nun auch die Initiatorinnen Stellung, siehe Villa Braslavsky/Geier (2020). 
So macht Merleau-Ponty darauf aufmerksam, dass wir nichts außer uns, sondern alles nur durch uns haben - vermittelt durch unsere Wahrnehmung. Dies bedeutet, dass uns die Welt durch uns selbst begegnet, weil sie uns ausschließlich durch unseren Leib vermittelt ist. Seine Philosophie, die mit sämtlichen empiristischen und intellektualistischen Ansätzen bricht, regt dazu an, die Vorstellung einer vollständig gegebenen Welt zu verwerfen und ihre „positive Unbestimmtheit“ (Waldenfels 2000: 70) anzuerkennen. Dabei geht es darum, die Welt im Werden und Entstehen „in statu nascendi“ (Merleau-Ponty 1966: 18) - zu begreifen. Waldenfels erläutert dies folgendermaßen:

„Die Welt selber entsteht [Herv. i. O.] auf gewisse Weise, sie ist nicht einfach fertig da als etwas, auf das wir uns umstandslos berufen können. Erfahrung bedeutet deshalb keine bloße Reproduktion fertiger Formen, mittels derer etwas registriert oder reproduziert wird, das in der Außenwelt oder im Geiste vorhanden wäre.“ (Waldenfels 2000: 63)

Das bedeutet auch, dass wir die Welt an sich nicht sehen können, da sie ihren Sinn immer erst durch Verweisung innerhalb eines Gesamtzusammenhang entfaltet. Dies wird im Zuge der Corona-Pandemie in zweierlei Hinsicht relevant: erstens in Bezug auf die Verschiebung des Ortes ohne die Anwesenheit der Anderen und zweitens in der Voraussetzung eines digitalen Endgerätes, um den Alltag an Hochschulen zu bewältigen.

Dies wird besonders deutlich, wenn wir aus der Perspektive der Leibphänomenologie Merleau-Pontys die körperliche Anwesenheit als ein Weltverhältnis verstehen, welches ich im Folgenden als leibliche Präsenz bezeichne. Dabei geht es nicht um die womöglich einhellige und dem Wortsinn leicht abgeleitete Einsicht eines simplen Daseins oder Nicht-Daseins als Form der Anwesenheit eines Körpers, sondern um Leiblichkeit als Ausgangspunkt menschlicher Existenz und Erfahrung. Die durch Gestalt und Struktur organisierte Wahrnehmung wird dann im Leib verortet. Der Leib als „affektive[r] Gegenstand“ (Merleau-Ponty 1966: 188) liegt dabei im Zentrum aller Orientierung und ermöglicht somit überhaupt erst eine Wahrnehmung von Raum und Zeit; er ist raumgreifend und stiftet ihn zugleich (vgl. Kristensen 2012: 24), wobei die räumliche Erfahrung immer in einer konkreten Situation stattfindet. Dies bedeutet, dass die nicht mehr zu realisierende körperliche Anwesenheit unsere Wahrnehmung der Lehr-, 
Lern- und Arbeitssituation verändert. ${ }^{4}$ Wir sitzen unter den veränderten Bedingungen nicht (mehr) in einem Hörsaal, einem Seminarraum, in der Mensa, im Büro, in Besprechungsräumen oder Fluren, sondern in Arbeitsund Schlafzimmern, Küchen und an Schreib- oder Esstischen.

Die Beobachtung der Relationierung und Kontextualisierung von Wahrnehmung einer konkreten Situation manifestiert sich am deutlichsten an dieser Verschiebung des Ortes: So bedeutet die Verlagerung der bisherigen Veranstaltungen ins Digitale eine Verschiebung in den privaten Raum. Nicht nur, dass nicht jede"r gleichermaßen digital ausgestattet ist, auch die räumlichen und sozialen Bedingungen des anderen Ortes sind nicht gleichermaßen und vor allem ausreichend gegeben. Der Rückzug, die Beschränkung und die Begrenzung auf die eigenen vier Wände haben dabei nicht nur eine soziale, sondern auch die eben beschriebene phänomenologische Dimension. So geht eine leiborientierte Phänomenologie davon aus, dass das „Erforschen und Bedenken der Dinge direkt oder indirekt immerzu auf den Leib und auf die leibliche Situation Bezug nimmt" (Waldenfels 2000: 9). Die leibliche Situation ist neben der räumlichen Veränderung zugleich von der körperlichen Abwesenheit der Anderen gekennzeichnet. Denn Leiblich-Sein bedeutet für Waldenfels sowohl ein Fürmich-Sein als auch ein Für-die-Anderen-Sein, sodass ein sehendes Wesen dabei zugleich auch immer ein Wesen ist, das gesehen wird (vgl. ebd.: 122). So kann angenommen werden, dass die Situation der Nicht-Präsenz der Anderen und von mir selbst an dem Ort der Hochschule das In-derWelt-Sein verändert. ${ }^{5}$

Deutlicher wird dies, wenn wir die Situation im Kontext des Digitalen betrachten. Nicht nur phänomenologisch kann das Wissen nicht von der Situation, in der es vermittelt, angeeignet, diskutiert und kritisiert wird, getrennt werden. Im Zusammenhang mit der digitalen Präsenz macht Waldenfels dabei auf folgendes aufmerksam: Die im Digitalen liegende Normierung von Erfahrung geschieht durch die Quantifizierung und Objektivierung von Interaktion und Wissenstransfer. So wurden aufgrund der fehlenden Anwesenheit die maßgeblichen Vorgänge an den Hochschulen

4 Die Soziologin Paula-Irene Villa Braslavsky schildert in einem Podcast ihr eigenes Unbehagen als lehrende Person während des Corona-Semesters wie folgt: „Leiblich-affektiv fühle ich mich von meiner eigenen Lehre wie abgeschnitten" (Villa Braslavsky/Geier 2020: 34:35-34:38). Ein wenig später fügt sie hinzu: „Trotzdem, wenn ich dieses bisherige Semester [...] Revue passieren lasse, da kommt es mir so vor als wäre ich gar nicht dabei gewesen“ (ebd.: 34:46-34:57).

5 Für die ausführlichere Diskussion der Folgen dieses veränderten Weltverhältnisses, siehe Abschnitt III. und IV. 
reorganisiert, Diskussionsformate in Chats und Foren verlegt, Zoom-Konferenzen terminiert oder Vorlesungen aufgezeichnet, um diese asynchron abrufen zu können. Dabei zeigt sich, dass die digitale Technik die Aufmerksamkeit auf die relevanten Inhalte formal aufrechterhalten kann. Die digitale Technologie kann sogar verschiedene Arten des Präsent-Seins, eine durch digitale Anwesenheit vermittelte Aufmerksamkeit ermöglichen (Ton an/aus, Bild an/aus, Chat- und Interaktionsfunktionen wie klatschen, melden, usw.). Durch Mimik, Gestik, Tonlage oder Aussprache können verschiedene Arten von Aufmerksamkeit artikuliert werden, sodass auch der oder die Andere in die eigenen Haltungen und Bewegungen einbezogen werden kann. Dennoch handelt es sich dabei stets um eine Reduktion der Situation, die - phänomenologisch gesprochen - der leiblichen Existenz des Menschen nicht gerecht wird (vgl. EAiR 2018).

Denn, so regt es die leiborientierte Phänomenologie an, wenn die leibliche Existenz des Menschen als Weltverhältnis begriffen wird, fungiert sie zugleich als Möglichkeitshorizont, der sich der Formalisierung und Reproduktion des Weltvollzugs entzieht und Erfahrung produzieren kann, die Unerwartetes und Überraschendes enthält. So ist es auch nicht verwunderlich, dass mit Waldenfels die Kommunikation mit digitalen Endgeräten ebenjene Formalisierung der Erfahrung von lernenden und lehrenden Subjekten bedeutet, nicht zuletzt weil die Formalisierung der Situation unerwartbare Erfahrungen und die Neustiftung von Sinn (auch in Abweichung) bestehender Ordnungen unwahrscheinlicher werden lässt (vgl. ebd.). Obgleich der Alltag an Hochschulen auch schon vor der Pandemie durch Formalisierung geprägt war, wie ich an späterer Stelle noch ausführen werde, verstärkt die fehlende leibliche Präsenz im Zuge der Pandemie diese Effekte.

\section{Präsenz als Bedingung des Einspruchs}

Diese phänomenologischen Einwände sollen nicht vorschnell zu der Annahme führen, dass die Anwesenheit an Hochschulen immer eine bessere Lehre und damit ein wahrhafteres Lernen und Arbeiten ermöglicht. Viel eher sollten sie den Blick auf die unterschiedlichen (räumlichen) Arrangements richten, die leibliche Präsenz ermöglichen bzw. verhindern. Darauf aufbauend soll der Blick in diesem zweiten Schritt auf die politische Dimension dieser als leiblich zu verstehenden Präsenz und die damit einhergehenden Möglichkeiten des von Judith Butler zur Sprache gebrachten Erscheinens gerichtet werden. 
In ihrem Werk Anmerkungen zu einer performativen Theorie der Versammlung (2016) macht sie auf das Phänomen des Erscheinens aufmerksam. In ihrer Auseinandersetzung mit temporären und kritischen Versammlungen wie den „Menschenmengen auf dem Tahrir-Platz in den Wintermonaten des Jahres 2010“ (Butler 2016: 7) deutet sie die darin liegenden Handlungen des Erscheinens als eine „verkörperte Form des Infragestellens der inchoativen und mächtigen Dimensionen herrschender Vorstellungen des Politischen“ (ebd.: 17). Auch wenn der Kontext, von dem aus Butler zu schreiben beginnt, ein anderer ist, sehe ich in ihren Überlegungen eine zentrale Anregung, die auch für die hier vorliegende Frage des Zusammenhangs von leiblicher Präsenz und Hochschulen relevant ist:

„Sich zeigen, stehen, atmen, sich bewegen, stillstehen, reden und schweigen, sind allesamt Aspekte einer plötzlichen Versammlung einer unvorhergesehenen Form politischer Performativität, die das lebenswerte Leben in den Vordergrund der Politik rückt. Und dies scheint schon der Fall zu sein, bevor die Gruppe damit begonnen hat, ihre Forderungen darzulegen oder sich im eigentlichen Sinne politisch zu äußern." (Butler 2016: 29)

Butler stellt einen Zusammenhang zwischen körperlicher Anwesenheit, verstanden als plurale Inszenierung, und dem Politischen her. Bereits das Erscheinen kann demnach politischen Charakter haben. Zugleich weist sie darauf hin, dass nicht jede Versammlung gleichsam demokratisch ist, und diskutiert dies anhand rechtspopulistischer Bewegungen wie Pegida (vgl. ebd.: 9f.). Ich möchte daran anschließend argumentieren, dass auch ich nicht jedwedes Erscheinen oder jede Versammlung von Körpern an den Hochschulen per se als politischen Akt oder politisch verstehe - mit Butler aber auf eine wichtige und interessante Verknüpfung von körperlicher Anwesenheit und politischer Artikulation hingewiesen werden kann. Anders als Butler, die auch von „virtuelle[n] und digitale[n] Netzwerken“ (ebd.: 16) ausgeht, in denen sich all jene Menschen versammeln, die nicht in „leiblicher Form erscheinen“ (ebd.: 16) können, argumentiere ich in Anschluss an den vorangegangenen Abschnitt zu Präsenz als Weltverhältnis für die materielle, affektive und leibliche Bedeutung des Erscheinens in nicht-digitalen Räumen. ${ }^{6}$ So nutze ich Butler an dieser Stelle, um auf die politische Bedeutung von Erscheinen als politischen Akt bzw. darüber hin-

6 Hier könnte eingewendet werden, dass Ereignisse wie die Anfänge des Arabischen Frühlings zeigen, dass durchaus politisches Potential in der Versammlung Vieler in virtuellen Netzwerken steckt. Dem würde ich entgegenhalten, dass Digitalität 
ausgehend auf dessen konstitutive Bedeutung für die politische Artikulation an Hochschulen hinzuweisen. Auch Butler tut dies, wenn sie an späterer Stelle schreibt, dass erst die Besetzung von Gebäuden, also deren Inanspruchnahme, an der Universität Berkeley die Diskussion über die Frage, ob Universitäten öffentliche oder private Institutionen sind, ermöglichte (vgl. ebd.: 128).

Butler geht dabei noch einen Schritt weiter, wenn sie über den Zusammenhang von versammelten Körpern und dem dazugehörigen Raum schreibt. So argumentiert sie, dass es zum einen materielle Voraussetzungen braucht, um zusammenzukommen, und zum anderen, dass die Materialität des Raumes durch die darin realisierte Versammlung und die Rede umgestaltet wird. Dabei bezieht sich Butler auch auf Hannah Arendts Auffassung über die Rechte der Versammlung und der freien Meinungsäußerung, die sie zugleich aufgrund der fehlenden Berücksichtigung körperlicher Aspekte im politischen Handeln zurückweist (vgl. Butler 2016: 99f.). Zentral für mich ist dabei aber der Aspekt, den Butler und Arendt gemeinsam vertreten, nämlich, dass Politik den Erscheinungsraum braucht und zugleich auch der Raum die Politik hervorbringt (vgl. ebd.: 100). Diese Reziprozität lässt sich auch auf den Raum der Hochschulen übertragen, der im Zuge der Corona-Pandemie - wenn auch nur temporär - keine Anwesenheit ermöglicht. Dies soll aber nicht darüber hinwegtäuschen, dass dieser durch die getroffenen politischen Entscheidungen maßgeblich zur Ermöglichung und Verhinderung von Erscheinen und dadurch politischem (Widerspruchs-)Handeln beiträgt. Dies soll im folgenden Abschnitt in den Blick genommen werden.

\section{Steuerung der Präsenz an Hochschulen}

Aufgrund der mit Butler skizzierten politischen Bedeutung, körperlich an einem Ort anwesend zu sein, rückt der Raum jener Institution in den Blick, die als unternehmerische Hochschule bezeichnet werden kann. ${ }^{7}$ Die Hochschulen sind seit Beginn der 1990er-Jahre einem tief- und weitreichenden Umbau unterworfen, den Alex Demirović in seinem Aufsatz Die

bzw. digitale Medien Kommunikationsprozesse vereinfachen, dass sich aber erst durch die körperliche Anwesenheit auf öffentlichen Plätzen in direkter Konfrontation mit der Materialität des Raumes (Regime, Exekutive, o.Ä.) Ordnungen angreifen, herausfordern und destabilisieren lassen.

7 Ähnliches beschreibt auch der Soziologe Richard Münch in Akademischer Kapitalismus. Über die politische Ökonomie der Hochschulreform (2011). 
Transformation der Staatlichkeit von Hochschulen (2007) im Hinblick auf folgende Merkmale kennzeichnet:

„Unternehmensförmige Steuerung der Hochschulen, stärkere Leistungsbewertung und -kontrolle der Hochschullehrer, eine (nun Modularisierung genannte) Verschulung der Studiengänge, Studiengebühren, Strafmaßnahmen für Langzeitstudierende, die Zwangsexmatrikulation, die Ersetzung des Abiturs durch Zugangs- und Auswahlgespräche, berufsbefähigende Abschlüsse nach sechs oder acht Semestern, die permanente Leistungskontrolle der Studierenden usw.“ (Demirović 2007: 537)

Diese strukturpolitischen Maßnahmen haben dabei im Kontext der Corona-Pandemie zwei relevante Folgen: zum einen die Verkürzung und zum anderen die Strukturierung von Anwesenheit an Hochschulen. Die Einführung der Bachelor- und Masterstudiengänge hat maßgeblich dazu beigetragen, die Zeitrhythmen der Studiendauer zu normieren und damit in letzter Konsequenz zu verkürzen. Dies bedeutet, dass der erste Studienabschluss häufig im Alter von 20 bis 22 Jahren erlangt wird, was zu einer sehr frühen „Verfügung über die Arbeitskraft und ihre kapitalistische Verwertung" (Demirović 2007: 539) führt. Nicht nur in der Frage von Zeit, sondern auch in der Form zeigt sich die beschriebene Transformation. Dazu zählt vor allem die Verschulung der Wissensvermittlung in der Ausbildung. Diese Formalisierung des Lernens und Lehrens hat auch die Durchführung eines digitalen Sommersemesters erst ermöglicht. ${ }^{8}$ So wurden bereits vor dem Ausbruch der Pandemie Leistung und Wissensfortschritt über die Errechnung des Workloads in die Abgabe von Studien- und Prüfungsleistungen übersetzt. Im Zuge der Corona-Pandemie erleichterte dieser Umstand die Umstellung ins Digitale, indem Leistung trotz fehlender Präsenz dergestalt gemessen und damit die Fortführung der Ausbildung ermöglicht werden kann.

Diese Entwicklung hin zu einer stärkeren Strukturierung des Aufenthalts an der Hochschule hat sich ebenfalls in den hochschulpolitischen Reaktionen auf die Krise gezeigt: Aus dieser Perspektive ist es nicht verwunderlich, dass zum einen die Forderungen des \#Nichtsemesters ${ }^{9}$ nur teilweise und schleppend von den Hochschulen aufgenommenen wurden und

8 Zugleich bleibt nur die Vermutung, dass das digitale Semester wiederum jene Effekte weiter verstärkt.

9 Weitere Informationen zum offenen Brief aus Forschung und Lehre, siehe Nichtsemester (2020). 
die finanzpolitischen Entscheidungen zur Unterstützung von Studierenden im Zuge der Corona-Pandemie, die unter dem Stichwort Nothilfe diskutiert und beschlossen wurden, nicht ausreichen, wie beispielsweise der freie zusammenschluss der student"innenschaften scharf kritisiert (vgl. Bühler 2020). ${ }^{10}$

Wenn also leibliche Präsenz als Bedingung des Einspruchs geltend gemacht werden kann, dann verhindern die hier mit Demirović skizzierten Entwicklungen - nicht nur im Zuge der Corona-Pandemie - im besonderen Maße Einspruch, Infragestellung und die „organisierte studentische Willensbildung und damit politische Erfahrungen“ (Demirović 2007: 543) an Hochschulen.

\section{Und wenn wir wieder da sind...}

Erst der Umstand der fehlenden körperlichen Anwesenheit an Hochschulen im Zuge der Corona-Pandemie scheint im Besonderen die Frage nach der Relevanz von Präsenz zu provozieren. Ausgehend von der Frage, was es eigentlich bedeutet, sich leiblich zu begegnen, wenn wir denken, lernen, lehren und arbeiten, verweist der Beitrag auf die phänomenologischen und politischen Dimensionen von Präsenz, die im Folgenden verbunden werden sollen.

So konnte zum einen gezeigt werden, dass in der Betrachtung der leiblichen Existenz des Menschen aus der Perspektive der Leibphänomenologie die Bedeutung von leiblicher Präsenz als Weltverhältnis an Hochschulen erkenntlich wird. Die durch die Digitalität ermöglichte Aufmerksamkeit im Zuge der Corona-Pandemie wird der leiblichen Existenz in Bezug auf ihr Weltverhältnis nicht gerecht. Dies ist vor allem in der hier skizzierten Perspektive der leiborientierten Phänomenologie problematisch, wenn wir uns noch einmal an Waldenfels erinnern: Mit ihm bedeutet leibliche Präsenz als Weltverhältnis eine Erfahrung, die keine bloße Reproduktion fertiger Formen darstellt, sondern die Neustiftung von Sinn und die Herausforderung von Ordnung durch ein leibliches, affektives und wahrnehmendes Subjekt ermöglicht.

10 Dabei sei an dieser Stelle auch an die prekäre Situation des akademischen Mittelbaus erinnert, dessen Befristungssituation ebenfalls als Festschreibung und Verkürzung einer zeitlichen Dimension von Präsenz verstanden werden kann. Dagegen hat sich unlängst auch eine politische Kampagne formiert, die als Frist ist Frust (2019) mehr unbefristete Stellen an Hochschulen fordert. 
Zum anderen verweisen sowohl Butler als auch Demirović auf die politische Bedeutung des Erscheinens und damit der körperlichen Anwesenheit als Bedingung des Einspruchs. So ermöglichen bzw. verhindern die Bedingungen des Raumes, in dem wir erscheinen (können), in erheblichen Maße Präsenz. Der von Demirović beschriebene strukturelle Umbau an den Hochschulen verdeutlicht diese Dynamik in besonderem Maße.

Für ein Lernen und Lehren, das sich als kritisch versteht, ist dies durchaus eine paradoxe Situation. Denn wenn wir leibliche Präsenz als die Grundbedingung für Kritik und politische Auseinandersetzung verstehen, um über die materiellen Bedingungen derselben streiten zu können, dann heißt das nichts anderes als: Wir müssen zusammenkommen, um über die Bedingungen, unter denen wir zusammenkommen, streiten zu können und sie im Zweifel in Frage zu stellen. In dieser Hinsicht ist Präsenz niemals nur der einfache Ausdruck der körperlichen Anwesenheit oder der digitalen Aufmerksamkeit, wie ich mit Butler, Waldenfels und Demirović in ihren phänomenologischen und politischen Analysen zeigen konnte. Vielmehr handelt es sich bei leiblicher Präsenz immer auch um die Erfahrung eines Weltverhältnisses, das bestehende (Sinn-)Ordnungen performativ und materiell herausfordert.

\section{Literaturverzeichnis}

Bühler, Jacob. 2020. Stellungnabme: Studierende während der epidemischen Lage nationaler Tragweite schützen. https://www.fzs.de/2020/05/25/stellungnahme-studieren de-waehrend-der-epidemischen-lage-nationaler-tragweite-schuetzen/. 12.06.2020.

Butler, Judith. 2016. Anmerkungen zu einer performativen Theorie der Versammlung. Berlin: Suhrkamp.

Demirović, Alex. 2007. Die Transformation der Staatlichkeit von Hochschulen. Das Argument 272: 531-545.

Dickel, Sascha. 2020. Gesellschaft funktioniert auch obne anwesende Körper. https://blo g.transcript-verlag.de/gesellschaft-funktioniert-auch-ohne-anwesende-koerper/. 12.06.2020.

EAiR. 2018. Akademiegespräche: Prof. Dr. Bernhard Waldenfels über Leiblichkeit und Digitalität. https://www.youtube.com/watch?v=mrQZAQPfV_o. 28.07.2020.

Frankfurter Arbeitskreis. 2020. Kritische Theorien in der Pandemie. Ein Glossar zur Corona-Krise. https://www.youtube.com/channel/UCqaO2Pg-95KgvH8pOO0h9 Dw/videos. 12.06.2020.

Frist ist Frust. 2019. Frist ist Frust. Entfristungspaket 2019. http://frististfrust.net. 28.07.2020. 
Kristensen, Stefan. 2012. Maurice Merleau-Ponty I. Körperschema und leibliche Subjektivät. In: Emmanuel Alloa et al. (Hrsg.), Leiblichkeit. Geschichte und Aktualität eines Konzeptes. Tübingen: UTB, 23-36.

Merleau-Ponty, Maurice. 1966. Phänomenologie der Wabrnehmung. Berlin: Walter de Gruyter.

Münch, Richard. 2011. Akademischer Kapitalismus, Über die politische Ökonomie der Hochschulreform. Frankfurt/M.: Suhrkamp.

Nichtsemester. 2020. \#nichtsemester. Ein offener Brief aus Forschung und Lehre. https:/ /www.nichtsemester.de/cbxpetition/offener-brief/. 28.07.2020.

Offener Brief. 2020. Zur Verteidigung der Präsenzlehre. https://www.praesenzlehre.co m. 12.06.2020.

Villa Braslavsky, Paula-Irene/Geier, Andrea. 2020. piqd Thema / BONUS: Die bessere Debatte: Präsenzlehre und Corona. https://detektor.fm/gesellschaft/piqd-thema-bo nus-die-bessere-debatte-praesenzlehre-und-corona?utm_campaign=twitter\&utm _ medium=twitter\&utm_source=twitter. 29.07.2020.

Waldenfels, Bernhard. 2000. Das leibliche Selbst. Vorlesungen zur Phänomenologie des Leibes. Frankfurt/M.: Suhrkamp. 
